\title{
Matrix abnormalities in pulmonary fibrosis
}

\author{
Chandak Upagupta, Chiko Shimbori, Rahmah Alsilmi and Martin Kolb
}

Affiliation: Firestone Institute for Respiratory Health, Dept of Medicine, McMaster University, Hamilton, ON, Canada.

Correspondence: Martin Kolb, Firestone Institute for Respiratory Health, Dept of Medicine, McMaster University, 50 Charlton Ave, Hamilton, ON, L8N 4AP, Canada. E-mail: kolbmamcmaster.ca

@ERSpublications

Cellular changes cause matrix aberrations that further drive cellular changes; this sustains and progresses fibrosis http://ow.ly/TpHq30kgEUX

Cite this article as: Upagupta C, Shimbori C, Alsilmi R, et al. Matrix abnormalities in pulmonary fibrosis. Eur Respir Rev 2018; 27: 180033 [https://doi.org/10.1183/16000617.0033-2018].

ABSTRACT Idiopathic pulmonary fibrosis (IPF) is a devastating, progressive disease, marked by excessive scarring, which leads to increased tissue stiffness, loss in lung function and ultimately death. IPF is characterised by progressive fibroblast and myofibroblast proliferation, and extensive deposition of extracellular matrix (ECM). Myofibroblasts play a key role in ECM deposition. Transforming growth factor (TGF)- $\beta 1$ is a major growth factor involved in myofibroblast differentiation, and the creation of a profibrotic microenvironment. There is a strong link between increased ECM stiffness and profibrotic changes in cell phenotype and differentiation. The activation of TGF- $\beta 1$ in response to mechanical stress from a stiff ECM explains some of the influence of the tissue microenvironment on cell phenotype and function. Understanding the close relationship between cells and their surrounding microenvironment will ultimately facilitate better management strategies for IPF.

\section{Introduction}

Idiopathic pulmonary fibrosis (IPF) is a chronic disease of unknown origin, which is characterised by progressive scarring of lung tissue, commonly in the peripheral (subpleural) lung parenchyma [1]. IPF is the most common form of idiopathic interstitial pneumonia and usually presents later in life. The median survival post-diagnosis is $\sim 3-4$ years, with a mortality rate that is higher than most common cancers $[2,3]$. Recently, two drugs have been approved for the management of IPF. Nintedanib and pirfenidone are both antifibrotic drugs, which have shown promise in reducing the decline of lung function and progression of IPF [4]. Although these drugs slow disease progression, they are not able to reverse or stop the structural damage and loss of lung function; therefore, the development of novel therapeutic targets for the treatment of pulmonary fibrosis is still of high priority.

The lung extracellular matrix (ECM) is comprised of collagens, elastin, glycoproteins and proteoglycans, which serve as structural scaffolding for cells, and also provide the mechanical stability and elastic recoil necessary for proper lung function. "Controlled" fibrogenesis is an indispensable wound healing response of the host to protect from injury. In normal wound healing, highly differentiated cells, called myofibroblasts, that produce ECM proteins are recruited during the active phase of repair, but are quickly removed via apoptosis once the repair process is underway. Most of the scar matrix subsequently resolves and is replaced by a more permanent tissue. In IPF however, myofibroblasts are relatively resistant to apoptosis, and their persistence leads to excessive scarring [5]. A hallmark of IPF is the accumulation of myofibroblasts in clusters called fibroblastic foci, and extensive ECM deposition within the interstitium

Provenance: Commissioned article, peer reviewed.

Received: March 272018 | Accepted after revision: May 292018

Copyright CERS 2018. ERR articles are open access and distributed under the terms of the Creative Commons Attribution Non-Commercial Licence 4.0. 
resulting in destruction of alveolar architecture. One hypothesis for the pathogenesis of IPF suggests that injury of alveolar epithelial cells is a key trigger; therefore, damaged epithelial cells can induce profibrotic factors and cause fibroblast proliferation and activation [6].

Through this continued fibroblast and myofibroblast proliferation and extensive ECM deposition, the parenchyma, epithelial barrier and alveolar architecture are destroyed. These structural changes are profibrotic, which further affects cell behaviour and accelerates fibrogenesis within the surrounding regions. This process is probably very similar among fibrotic lung diseases of different origin, and not only applicable to IPF [7]. Patients experience this as a relentless decline in lung function, and ultimately respiratory failure and death. In this brief review, we discuss the matrix abnormalities in pulmonary fibrosis and how they lead to lung stiffening and organ dysfunction.

\section{Mechanical stiffness in pulmonary fibrosis}

The ECM of fibrotic lungs has abnormal biochemical and biomechanical characteristics. Stiffening of the lung parenchyma causes shortness of breath, the first clinical symptom of IPF [8]. Increasing lung stiffness is indirectly measured by the decline in forced vital capacity (FVC), which correlates with mortality and is the best parameter to determine IPF progression [9]. One possible relationship between stiffness and the peripheral and basal fibrosis pattern seen in IPF has been proposed using a mathematical model of lung maximal stiffness, which suggests that the distribution of IPF lesions corresponds to the areas of greatest mechanical distension during breathing [10]. Compliance, a more direct parameter of stiffness, measures the elastic properties of the lung. Reduced lung compliance is observed in IPF patients, and compliance constantly declines throughout the course of the disease [11]. Compliance is typically measured in rodents with experimental models of lung fibrosis [12]. Increased stiffness is also determined in fibrotic animal lungs using atomic force microscopy and Young's modulus measurements $[13,14]$. Changes in tissue stiffness seem to precede the fibrotic response, which has also been seen in the context of liver fibrosis [15]. This relationship is probably also present in IPF lungs, where increased stiffness is a significant contributor to disease progression.

\section{Transforming growth factor- $\beta 1$ in ECM remodelling and pulmonary fibrosis}

Transforming growth factor (TGF)- $\beta$ is a growth factor known to regulate numerous essential cellular processes such as cell growth, proliferation, differentiation and apoptosis [16]. Three major mammalian isoforms of this growth factor have been identified: TGF- $\beta 1$, TGF- $\beta 2$ and TGF- $\beta 3$, with TGF- $\beta 1$ being the most closely related to the development of pulmonary fibrosis $[17,18]$. TGF- $\beta$ is a secreted protein that is synthesised by many parenchymal and infiltrating cells. It is stored in the ECM, in an inactive form bound within the small latent complex (figure 1) [19]. TGF- $\beta 1$ content and activity is increased within the lungs of experimental fibrosis models and IPF [20, 21]. TGF- $\beta 1$ overexpression induces persistent pulmonary fibrosis in rodents via the canonical Smad3 signalling pathway. Genetic deletion of Smad3 protects mice from TGF- $\beta 1$ - and bleomycin-induced pulmonary fibrosis [17, 22].

TGF- $\beta 1$ mediates many fibrogenic responses, including myofibroblast activation and differentiation [23]. TGF- $\beta 1$ also regulates the epithelial-mesenchymal transition, a phenotypic change in which epithelial cells acquire mesenchymal characteristics [24]. Myofibroblasts can originate from a variety of precursor cells, such as resident fibroblasts, endothelial cells, pericytes and fibrocytes, and TGF- $\beta 1$ plays a major role in the transdifferentiation of all these cell types [25-27]. Mesenchymal stem cells have also been seen to undergo myofibroblast-like differentiation in response to TGF- $\beta 1$ [28]. In addition to myofibroblast differentiation, TGF- $\beta 1$ also regulates the characteristics of the other cell types. For example, TGF- $\beta 1$ promotes M2 macrophage polarisation, a known profibrotic phenotype that produces more TGF- $\beta 1$ [29]. Altogether, it is clear that TGF- $\beta 1$ plays a key role in ECM composition and homeostasis, and hence the development and exponential progression of pulmonary fibrosis.

As mentioned previously, myofibroblasts are rarely found in healthy human physiology; however, they become vastly upregulated after injury and are crucial for proper wound healing [30]. Myofibroblasts possess bundles of microfilaments which reach the cell surface in a specialised adhesion complex, termed the "fibronexus" or mature local adhesion. This complex bridges myofibroblasts' internal microfilaments with extracellular fibronectin domains, thus functioning as a contractile link that enables these cells to generate a contractile force on the surrounding ECM. In tissues with increased stiffness, these mechanisms are obviously magnified.

\section{Mechanical stress induces TGF- $\beta 1$ activation}

One suggested mechanism to explain the influence of increased mechanical stiffness on cell phenotype and characteristic is the force-induced activation of TGF- $\beta 1$. Previous in vitro studies have suggested that mechanical stiffness contributes to TGF- $\beta 1$ activation [31]. Recent experiments performed by our group 


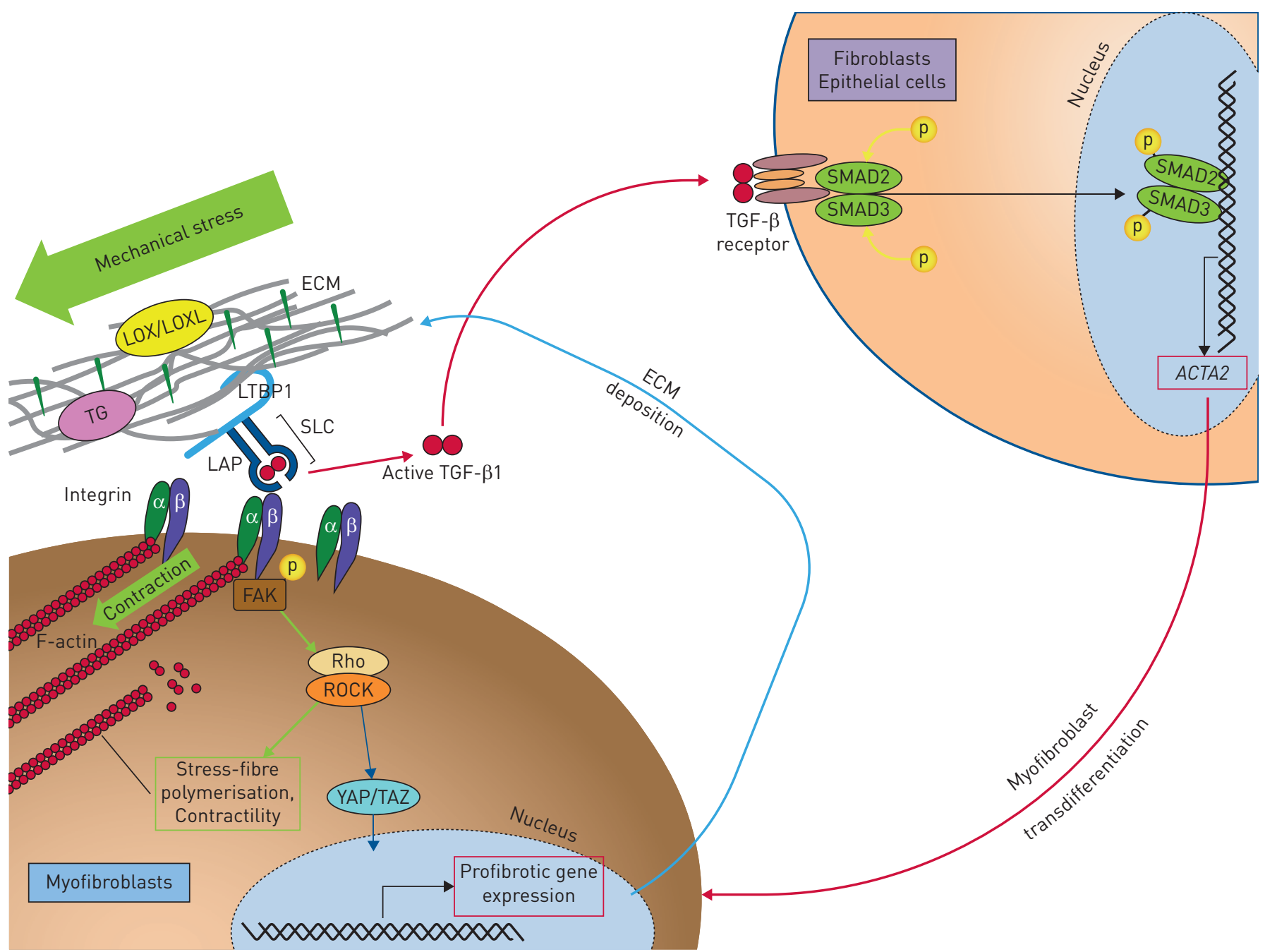

FIGURE 1 The dynamic interplay between the lung extracellular matrix (ECM) and resident cells, with transforming growth factor (TGF)- $\beta 1$ at its centre. The active TGF- $\beta 1$ protein in red remains trapped within the latency associated peptide (LAP) to form the small latent complex (SLC). The SLC is attached to the ECM via the latent TGF- $\beta$ binding protein (LTBP). Integrins found on the surface of cells link the LAP with the cell's cytoskeleton to allow for the transduction of the mechanical signal within the cell. In addition, the integrin attaches to the LAP and serves as an anchor; upon mechanical stress the SLC is cleaved and releases the bioactive TGF- $\beta 1$ homodimer. The active TGF- $\beta 1$ protein is then able to bind to the TGF- $\beta 1$ receptor located on neighbouring cells, i.e. fibroblasts/epithelial cells. The activation of this receptor results in the phosphorylation of SMAD2/3, causing its translocation to the nucleus where it promotes the transcription of ACTA2 (the mRNA for $\alpha$-SMA), resulting in the differentiation of these cells into myofibroblasts. The cyclical relationship between TGF- $\beta 1$ activation via myofibroblasts and the resulting myofibroblast differentiation can be visualised through the red arrows. Mechanical stress also causes contraction of F-actin via integrins, which results in the phosphorylation of focal adhesion kinase (FAK) to promote further actin polymerisation. FAK phosphorylation also activates Rho kinase (ROCK) via complexing with RhoA, which results in increased actomyosin contractility. This cyclical relationship of cell contractility promoting further stress-fibre polymerisation and contractility is outlined by the bright green arrows. In response to these mechanical signals, the mechanosensitive proteins YAP (yes-associated protein) and TAZ (transcriptional coactivator with PDZ-binding motif) are translocated into the nucleus of the cell to promote the transcription of profibrotic genes. Genes such as collagen and other ECM proteins are produced, promoting ECM deposition. This causes the cells' surroundings to become rigid and stiff, therefore further adding to the mechanical stress placed on the cells (indicated by the blue arrow). TG: transglutaminase; LOX/LOXL: lysyl oxidase/lysyl oxidase-like.

demonstrated that mechanical stretch activates and releases latent TGF- $\beta 1$ in living tissues from fibrotic lungs (rat and human) (figure 1) [13]. In this study, fibrotic and normal lung strips were subjected to cyclic mechanical stimuli in a tissue bath equipped with a force transducer and servo-controlled arm to explore the effect of mechanical stress on growth factor activation. The activation and release of TGF- $\beta 1$ from the lung strips were measured in the tissue bath solutions. We determined that the mechanical stress induced active TGF- $\beta 1$ release and also activated TGF- $\beta 1$ downstream signalling pathways in the fibrotic lungs [13]. While protease and protein secretion inhibitors did not change mechanical stretch-induced TGF- $\beta 1$ activation or Smad2/3 phosphorylation, TGF- $\beta 1$ receptor inhibitors (SD208) did. Furthermore, the induction of phospho-Smad2/3 was enhanced in whole fibrotic rat lungs undergoing ventilation pressure challenge, compared with control lungs [13]. Interestingly, the tissue stiffness, as measured by 
increased Young's modulus, was positively correlated with the amount of active TGF- $\beta 1$ released by the tissues following mechanical stretch [13]. These findings suggest that the stiffness of the surrounding environment is capable of activating TGF- $\beta 1$, which may play a crucial role in the ability of the ECM to dictate a profibrotic cell phenotype, and promote progressive pulmonary fibrosis.

\section{Mechanotransduction and pulmonary fibrosis}

The ECM stiffness itself may contribute to IPF development, irrespective of the cyclical stretch that occurs during breathing. Therefore, targeting ECM stiffness and the following mechanotransduction pathway is a promising target for IPF therapy. External forces applied to cells lead to the clustering of integrins to form aggregates (e.g. in focal adhesions), which are sites for the transfer of mechanical force from the ECM to the cytoskeleton. These integrins activate Rho kinase (ROCK) and phosphorylation of focal adhesion kinase (FAK) which promotes actin polymerisation [32]. Activated ROCK and FAK induce activation of the myosin light chain (MLC) as well as inhibition of MLC phosphatase, abruptly increasing myosin-II activity and actomyosin contractility. These changes collectively translate into profibrotic phenotypic changes in fibroblasts (figure 1). In vitro studies have shown that ROCK and FAK potently stimulate the differentiation of fibroblasts into myofibroblasts $[33,34]$. Our tissue stretch study determined that a ROCK inhibitor, Y-27632, inhibited mechanical stretch-induced TGF- $\beta 1$ activation. Similarly, the ROCK inhibitor fasudil demonstrated a therapeutic effect in the pulmonary fibrosis model [13, 35], as did a FAK inhibitor [36]. The mechanosensitive proteins YAP (yes-associated protein) and TAZ (transcriptional coactivator with PDZ-binding motif) also regulate fibroblast function. Furthermore, overexpression of YAP or TAZ has been shown to induce pulmonary fibrosis in murine lungs [37]. Together, these results highlight that the mechanotransduction pathways are a key step in fibrogenesis, and may be attractive as targets in fibrosis therapies.

\section{ECM cross-linking enzymes in pulmonary fibrosis}

With respect to lung structure, cross-linking enzymes play an important role in the ECM homeostasis. The biomechanical properties of the ECM are strongly influenced by the biochemical composition of the matrix and its individual components, and also by post-translational modifications, such as glycosylation, transglutamination and cross-linking [38]. These cross-linking enzymes, including prolyl hydroxylase, transglutaminases (TGs), and lysyl oxidase (LOX) and its lysyl oxidase-like (LOXL1-4) congeners, regulate the stability, maturation and turnover of collagen, and orient the ECM assembly for correct function [39]. Interestingly, hypoxic conditions increase the secretion of LOX, LOXL2 and LOXL4 from normal human fibroblasts in vitro [40]. Since hypoxic conditions are found within highly fibrotic regions of the IPF lung, it is important to better understand the role hypoxia plays on cross-linking enzymes and fibrosis. Neutralising antibodies against LOXL2 inhibited a number of fibrotic states, including that of bleomycin-induced pulmonary fibrosis [41]. Higher serum LOXL2 levels in patients with IPF were associated with an increased risk of disease progression [42]. Furthermore, recent experiments performed by our group in LOXL1-deficient mice clearly showed that the absence of this enzyme protected against pulmonary fibrosis, via reduced collagen cross-linking and decreased tissue stiffness [43]. Similarly, mice deficient in TG2, another important cross-linking enzyme, were protected from bleomycin-induced pulmonary fibrosis $[32,44]$. A recent study investigating the role of both TG and LOXL enzymes concluded that the pathological cross-linking of the ECM in IPF increases fibroblast growth and resistance to normal ECM turnover [45]. These results suggest that cross-linking enzymes regulate pulmonary fibrosis development, and that cross-linking could be a reasonable target for decreasing stiffness and stopping the mechanical stretch-induced profibrotic response. Despite the promising experimental studies, a recent phase II clinical trial targeting LOXL2, with the LOXL2 inhibitor simtuzumab, did not show an effect on progression-free survival in patients with IPF for reasons that are not yet clearly understood [46]. This negative trial result highlights the need for further research into understanding the complex mechanism involving LOXL and other cross-linking enzymes in IPF.

\section{The ECM proteome in pulmonary fibrosis}

The ECM is comprised of more than 300 proteins that make up the "matrisome core". Polysaccharides, glycosaminoglycans, ECM-binding growth factors and ECM-modifying enzymes are attached to this core, and together they serve as the tissue scaffold. The ECM structure is highly dynamic and is constantly enzymatically and non-enzymatically remodelled. In IPF, this remodelling is dysregulated with imbalances in protein secretion, degradation, and organisation. This results in the hallmarks of IPF: increased deposition of proteoglycans, collagen, elastin and fibronectin. Proteoglycans consist of a core protein that binds to a highly charged glycosaminoglycan. Together they make up the integral hydrated gel, which binds collagen and elastin fibres to provide mechanical stability for the alveolar structure. Dysregulated remodelling of the lung causes abnormal expression of the proteoglycan-glycosaminoglycan complexes and leads to the destruction of the architecture [47]. Fibrillar collagen is the most abundant type of 
collagen in the lung and is crucial for tensile strength and functionality. In IPF, collagen accumulates around myofibroblasts within fibroblastic foci resulting in increased stiffness within these regions [48]. Elastin fibres attach to collagen and contribute to the lung's elastic recoil. Elastin is increased in bleomycin-treated mouse lungs [49]. Elastin has also been seen to enhance TGF- $\beta 1$-induced myofibroblast differentiation. Glycoproteins, such as fibronectin, are responsible for mediating cell-matrix adhesions by binding ECM proteins such as collagen and cell-surface integrins. Fibronectin and other glycoproteins are found in abundance in the lungs of IPF patients [50]. Similar to elastin, fibronectin is also essential in driving myofibroblast differentiation. These findings suggest that the compositional changes of the fibrotic ECM alone can drive a profibrotic cell phenotype. Therefore, proteomic studies are needed to better understand the dynamics of the matrix, and to improve ECM diagnostics to develop treatments for fibrotic diseases.

\section{Conclusion}

ECM stiffening is associated with the presentation of clinical features and progressive decline of lung function in IPF. The recruitment and accumulation of myofibroblasts plays a major role in aberrant tissue remodelling and fibrosis. The intrinsic role that the ECM plays in driving progressive fibrosis on its own is incompletely understood. Mechanical stretch related to breathing can cause activation of one of the key fibrogenic mediators: TGF- $\beta 1$. Targeting the connection between structural cells in the lung interstitium (myofibroblasts) and their microenvironment (lung matrix) is a promising area for development of novel intervention strategies to improve IPF. This can be achieved by targeting intracellular, extracellular or membrane-bound molecules that are all involved in this process.

Conflict of interest: M. Kolb reports a research grant from Intermune/Roche Canada, during the conduct of the study. $\mathrm{He}$ also reports grants and personal fees from Roche, Boehringer Ingelheim and GSK, personal fees from Gilead, Prometic and Genoa, and grants from Actelion, Respivert, Alkermes and Pharmaxis, outside the submitted work. C. Upagupta reports grants from the Canadian Institute for Health Research, during the conduct of the study. C. Shimbori reports grants from the Canadian Institute for Health Research, during the conduct of the study.

Support statement: Funding support was received from the Canadian Institute for Health Research, Institute of Circulatory and Respiratory Health. Funding information for this article has been deposited with the Crossref Funder Registry.

\section{References}

1 Selman M, Pardo A. Revealing the pathogenic and aging-related mechanisms of the enigmatic idiopathic pulmonary fibrosis. An integral model. Am J Respir Crit Care Med 2014; 189: 1161-1172.

2 Vancheri C, Failla M, Crimi N, et al. Idiopathic pulmonary fibrosis: a disease with similarities and links to cancer biology. Eur Respir J 2010; 35: 496-504.

3 Ley B, Collard HR, King TE. Clinical course and prediction of survival in idiopathic pulmonary fibrosis. Am J Respir Crit Care Med 2011; 183: 431-440.

4 Raghu G, Selman M. Nintedanib and pirfenidone. New antifibrotic treatments indicated for idiopathic pulmonary fibrosis offer hopes and raises questions. Am J Respir Crit Care Med 2015; 191: 252-254.

5 Bellaye P-S, Kolb M. Why do patients get idiopathic pulmonary fibrosis? Current concepts in the pathogenesis of pulmonary fibrosis. BMC Med 2015; 13: 176

6 Selman M, Pardo A. Alveolar epithelial cell disintegrity and subsequent activation: a key process in pulmonary fibrosis. Am J Respir Crit Care Med 2012; 186: 119-121.

7 Wells AU, Brown KK, Flaherty KR, et al. What's in a name? That which we call IPF, by any other name would act the same. Eur Respir J 2018; 51: 1800692.

8 Daniels CE, Ryu JH. Treatment of idiopathic pulmonary fibrosis. Semin Respir Crit Care Med 2006; 27: 668-676.

9 Martinez FJ, Flaherty K. Pulmonary function testing in idiopathic interstitial pneumonias. Proc Am Thorac Soc 2006; 3: 315-321.

10 Carloni A, Poletti V, Fermo L, et al. Heterogeneous distribution of mechanical stress in human lung: a mathematical approach to evaluate abnormal remodeling in IPF. J Theor Biol 2013; 332: 136-140.

11 Zielonka T, Demkow U, Radzikowska E, et al. Angiogenic activity of sera from interstitial lung disease patients in relation to pulmonary function. Eur J Med Res 2010; 15: Suppl. 2, 229-234.

12 Ask K, Labiris R, Farkas L, et al. Comparison between conventional and "clinical" assessment of experimental lung fibrosis. J Transl Med 2008; 6: 16.

13 Froese AR, Shimbori C, Bellaye P-S, et al. Stretch-induced activation of transforming growth factor- $\beta 1$ in pulmonary fibrosis. Am J Respir Crit Care Med 2016; 194: 84-96.

14 Liu F, Mih JD, Shea BS, et al. Feedback amplification of fibrosis through matrix stiffening and COX-2 suppression. J Cell Biol 2010; 190: 693-706.

15 Georges PC, Hui J-J, Gombos Z, et al. Increased stiffness of the rat liver precedes matrix deposition: implications for fibrosis. Am J Physiol Gastrointest Liver Physiol 2007; 293: G1147-G1154.

16 Border WA, Noble NA. Transforming growth factor beta in tissue fibrosis. N Engl J Med 1994; 331: 1286-1292.

17 Sime PJ, Xing Z, Graham FL, et al. Adenovector-mediated gene transfer of active transforming growth factor- $\beta 1$ induces prolonged severe fibrosis in rat lung. J Clin Invest 1997; 100: 768-776.

18 Xaubet A, Marin-Arguedas A, Lario S, et al. Transforming growth factor- $\beta 1$ gene polymorphisms are associated with disease progression in idiopathic pulmonary fibrosis. Am J Respir Crit Care Med 2003; 168: 431-435. 
19 Leppäranta O, Sens C, Salmenkivi K, et al. Regulation of TGF- $\beta$ storage and activation in the human idiopathic pulmonary fibrosis lung. Cell Tissue Res 2012; 348: 491-503.

20 Westergren-Thorsson G, Hernnäs J, Särnstrand B, et al. Altered expression of small proteoglycans, collagen, and transforming growth factor- $\beta 1$ in developing bleomycin-induced pulmonary fibrosis in rats. J Clin Invest 1993; 92: 632-637.

21 Khalil N, O'Connor RN, Unruh HW, et al. Increased production and immunohistochemical localization of transforming growth factor- $\beta$ in idiopathic pulmonary fibrosis. Am J Respir Cell Mol Biol 1991; 5: 155-162.

22 Bonniaud P, Kolb M, Galt T, et al. Smad3 null mice develop airspace enlargement and are resistant to TGF- $\beta$-mediated pulmonary fibrosis. J Immunol 2004; 173: 2099-2108.

23 Evans RA, Tian YC, Steadman R, et al. TGF- $\beta 1$-mediated fibroblast-myofibroblast terminal differentiation-the role of Smad proteins. Exp Cell Res 2003; 282: 90-100.

24 Xu J, Lamouille S, Derynck R. TGF- $\beta$-induced epithelial to mesenchymal transition. Cell Res 2009; 19: 156-172.

25 Piera-Velazquez S, Li Z, Jimenez SA. Role of endothelial-mesenchymal transition (EndoMT) in the pathogenesis of fibrotic disorders. Am J Pathol 2011; 179: 1074-1080.

26 Schmidt M, Sun G, Stacey MA, et al. Identification of circulating fibrocytes as precursors of bronchial myofibroblasts in asthma. J Immunol 2003; 171: 380-389.

27 Chen Y-T, Chang F-C, Wu C-F, et al. Platelet-derived growth factor receptor signaling activates pericyte-myofibroblast transition in obstructive and post-ischemic kidney fibrosis. Kidney Int 2011; 80: 1170-1181.

28 Mishra PJ, Banerjee D. Activation and Differentiation of Mesenchymal Stem Cells. In: Kalyuzhny AE, ed. Signal Transduction Immunohistochemistry. Totowa, Humana Press, 2011; pp. 245-253. Available from: http://link. springer.com/10.1007/978-1-61779-024-9_14

29 Gong D, Shi W, Yi S, et al. TGF $\beta$ signaling plays a critical role in promoting alternative macrophage activation. BMC Immunol 2012; 13: 31.

30 Gabbiani G. The myofibroblast in wound healing and fibrocontractive diseases. J Pathol 2003; 200: 500-503.

31 Wipff P-J, Rifkin DB, Meister J-J, et al. Myofibroblast contraction activates latent TGF- $\beta 1$ from the extracellular matrix. J Cell Biol 2007; 179: 1311-1323.

32 Janoštiak R, Pataki AC, Brábek J, et al. Mechanosensors in integrin signaling: the emerging role of p130Cas. Eur J Cell Biol 2014; 93: 445-454.

33 Greenberg RS, Bernstein AM, Benezra M, et al. FAK-dependent regulation of myofibroblast differentiation. FASEB J 2006; 20: 1006-1008.

34 Akhmetshina A, Dees C, Pileckyte M, et al. Rho-associated kinases are crucial for myofibroblast differentiation and production of extracellular matrix in scleroderma fibroblasts. Arthritis Rheum 2008; 58: 2553-2564.

35 Zhou Y, Huang X, Hecker L, et al. Inhibition of mechanosensitive signaling in myofibroblasts ameliorates experimental pulmonary fibrosis. J Clin Invest 2013; 123: 1096-1108.

36 Kinoshita K, Aono Y, Azuma M, et al. Antifibrotic effects of focal adhesion kinase inhibitor in bleomycin-induced pulmonary fibrosis in mice. Am J Respir Cell Mol Biol 2013; 49: 536-543.

37 Liu F, Lagares D, Choi KM, et al. Mechanosignaling through YAP and TAZ drives fibroblast activation and fibrosis. Am J Physiol Lung Cell Mol Physiol 2015; 308: L344-L357.

38 Erler JT, Weaver VM. Three-dimensional context regulation of metastasis. Clin Exp Metastasis 2009; 26: 35-49.

39 Kadler KE, Hill A, Canty-Laird EG. Collagen fibrillogenesis: fibronectin, integrins, and minor collagens as organizers and nucleators. Curr Opin Cell Biol 2008; 20: 495-501.

40 Aumiller V, Strobel B, Romeike M, et al. Comparative analysis of lysyl oxidase (like) family members in pulmonary fibrosis. Sci Rep 2017; 7: 149.

41 Barry-Hamilton V, Spangler R, Marshall D, et al. Allosteric inhibition of lysyl oxidase-like-2 impedes the development of a pathologic microenvironment. Nat Med 2010; 16: 1009-1017.

42 Chien JW, Richards TJ, Gibson KF, et al. Serum lysyl oxidase-like 2 levels and idiopathic pulmonary fibrosis disease progression. Eur Respir J 2014; 43: 1430-1438.

43 Bellaye P-S, Shimbori C, Upagupta C, et al. Lysyl oxidase-like 1 protein deficiency protects mice from adenoviral transforming growth factor- $\beta 1$-induced pulmonary fibrosis. Am J Respir Cell Mol Biol 2018; 58: 461-470.

44 Olsen KC, Sapinoro RE, Kottmann RM, et al. Transglutaminase 2 and its role in pulmonary fibrosis. Am J Respir Crit Care Med 2011; 184: 699-707.

45 Philp CJ, Siebeke I, Clements D, et al. Extracellular matrix cross-linking enhances fibroblast growth and protects against matrix proteolysis in lung fibrosis. Am J Respir Cell Mol Biol 2018; 58: 594-603.

46 Raghu G, Brown KK, Collard HR, et al. Efficacy of simtuzumab versus placebo in patients with idiopathic pulmonary fibrosis: a randomised, double-blind, controlled, phase 2 trial. Lancet Respir Med 2017; 5: 22-32.

47 Kulkarni T, O’Reilly P, Antony VB, et al. Matrix remodeling in pulmonary fibrosis and emphysema. Am J Respir Cell Mol Biol 2016; 54: 751-760.

48 Kuhn C, Boldt J, King TE, et al. An immunohistochemical study of architectural remodeling and connective tissue synthesis in pulmonary fibrosis. Am Rev Respir Dis 1989; 140: 1693-1703.

49 Blaauboer ME, Boeijen FR, Emson CL, et al. Extracellular matrix proteins: a positive feedback loop in lung fibrosis? Matrix Biol 2014; 34: 170-178

50 Serini G, Bochaton-Piallat ML, Ropraz P, et al. The fibronectin domain ED-A is crucial for myofibroblastic phenotype induction by transforming growth factor-beta1. J Cell Biol 1998; 142: 873-881. 